\title{
Evaluation of Expected Software Quality: A Customer's Viewpoint
}

\author{
Krzysztof Sacha \\ Warsaw University of Technology, Nowowiejska 15/19, \\ Warszawa 00-665, Poland \\ k.sacha@ia.pw.edu.pl
}

\begin{abstract}
The paper describes an approach, which we developed to assess the expected quality of software for a huge governmental system. The assessment was done on behalf of the customer, and not of the development company, and was performed within two years along with the software development process. Our approach was based on a modification to GQM and was focused on the evaluation of the quality of methods and the deliverables of the project. The paper describes the areas of the quality evaluation, the questions that we stated and the metrics that we used. The statistical metrics defined in ISO 9126 appeared not very helpful within the context of this project.
\end{abstract}

\section{Introduction}

Software engineering is founded upon a set of paradigms, technologies and processes that enable the disciplined development and evolution of software systems. As with any other engineering discipline, software engineering requires methods and measurement mechanisms for evaluation of the quality of software products. Because the software development process is long and expensive, those methods and mechanisms should not only allow for the evaluation of the quality of an existing software system but also the expected quality of product under design.

Effective software quality evaluation and assurance requires models that describe what the software quality is and how can it be traced back to the development process. Two different approaches to software quality have been defined recently in a set of international ISO standards. One is focused on assuring high quality of the process by which the product is developed, while the other is aimed at a direct definition of the attributes and metrics that characterize the quality of the software product.

The requirements for a quality management system are defined in ISO 9001 [1]. All the requirements are intended for application within a software process in order to enhance the customer satisfaction, which is considered the primary measure of the software product quality. The quality management system, as defined by the standard, can be subject to a certification.

Quality characteristics of the software product are defined in ISO 9126 [2]. The characteristics are subdivide into attributes that can be measured by means of appropriate metrics. A set of metrics is defined in the accompanied technical reports [3-5]. Such definitions help in evaluating the quality of an existing software system, but gives no guidance on how to construct a high quality software product. 
Unfortunately, the two standards have not been related to each other in that the quality characteristics of ISO 9126 are not referenced by ISO 9001.

A practical application of the recommendations of both of the two ISO standards needs a method for selecting the metrics and collecting data that are relevant for a particular purpose. There are a few such methods described in the literature, with the Goal Question Method approach [6-8] and the Quality Function Deployment approach [9-11] being the best known examples. All of those methods represent the viewpoint of the software development organization.

This paper describes a method, which we used to assess the expected quality of a huge software system that was developed in the years of 2003-2004 to support European Union's Common Agriculture Policy in Poland. IACS (Integrated Administration and Control System) was built in time and deployed in more than 300 regional offices. Currently, the system processes data from more than 2000000 farms.

The assessment was performed along with the software development process and was focused on the evaluation of the quality of methods and deliverables of the particular steps of the project. Our approach was based on a modification to Goal Question Metric approach. The modification was needed, because our assessment was done on behalf of the customer, and not of the development company, and it had no other goal in mind than just to evaluate the expected quality of the developed software. Because our customer requested full compliance with the requirements that could not be compromised, the statistical metrics defined in ISO 9126 appeared inadequate within the environment of this project.

The paper is organized as follows. Section 2 provides the reader with a short overview of the approach represented by ISO 9001, and Section 3 summarizes the approach of ISO 9126. The method that we used to evaluate the quality of the development of the IACS software is presented in Section 4, and the set of detailed questions and metrics used by the method is described in Section 5. Final remarks are gathered in Conclusions.

\section{ISO 9001 Overview}

ISO 9001 [1] describes the requirements for a quality management system, which is a part of the total manufacturing process. The standard is very general and applies to all types of organizations, regardless of their size and of what they do. The recommended practices can help both product and service oriented organizations and, in particular, can be used within the context of software development and manufacturing. ISO 9001 certificates are recognized and respected throughout the world.

Because of this generality it is not easy to map the recommendations of the standard into the practical activities that can be performed within a software process. Moreover, the standard is intended to be used by the manufacturers and not by the auditors that work on behalf of their customers. Therefore, it contains many recommendations that relate to resource management process, which was completely outside the scope of our evaluation. What we were expected to assess was the quality of the methods that were used by the manufacturer throughout the software development process and the quality of products of particular steps of the development: Analytical specifications, design documents, test plans and procedures, user manuals and the 
resulting code. The actual implementation of code and of the testing process was also subject to our evaluation.

ISO 9001 does not define any particular model of quality. Instead, it adopts a simple approach that the quality of a product is measured by the customer satisfaction. According to this approach, no quality characteristics are defined, and the only basis for quality evaluation are the customer requirements. If those requirements are met, then the product quality can be evaluated high. The lack of a quality model makes this standard orthogonal to ISO 9126. There are no common points between the two, but also no contradiction can be found.

The top level requirement of ISO 9001 is such that a quality management system must be developed, implemented and maintained. All the processes and activities performed within the scope of this system have to be documented and recorded for the purpose of future review. A huge part of the standard relates to the processes of quality planning and management, resource management, and continuous quality monitoring, analysis and improvement. This part is not very helpful in evaluating the quality of a specific software product under design.

The part, which relates directly to the body of a software project, is a section on realization requirements. Basic requirements and recommendations that are stated therein can be summarized as follows:

1. Identify customer's product requirements, i.e. the requirements that the customer wants to meet, that are dictated by the product's use or by legal regulations.

2. Review the product requirements, maintain a record of the reviews, and control changes in the product requirements.

3. Develop the software process, clarify the responsibilities and authorities, define the inputs and outputs of particular stages.

4. Perform the necessary verification and validation activities, maintain a record of these activities, and manage design and development changes.

All of those statements are very concrete and provide valuable guidelines for auditing and evaluating the quality of a software process. Moreover, the stress that is placed on the need to meet customer requirements helps in closing the gap between the quality of the software process and the quality of software itself.

\section{ISO/IEC 9126 Overview}

ISO 9126 [2] is concerned primarily with the definition of a quality model, which can be used to specify the required product quality, both for software development and software evaluation. The model defines three different views of the software quality:

- Quality in use view captures the ability of a software product to help the user in achieving his or her specific goals within the specified context of use.

- External quality view captures the characteristics of a software product that can be observed when the software is executed.

- Internal quality view captures the characteristics of a software product that can be measured based on intermediate products during the software development process. 
The views are related to each other in such a way that quality in use characteristics depend on the external quality characteristics, which in turn depend on the internal quality characteristics. Only the internal quality characteristics can be observed during the development process and used in order to predict the external quality and the quality in use of the final software product.

The internal and external quality models share the same set of six characteristics, which are intended to be exhaustive. All the six quality characteristics, defined in ISO 9126, are recapitulated below, along with some comments.

Functionality is defined as the ability of the software product to provide functions which meet stated or implied needs of the user. This is a very basic characteristic, which is semantically close to the property of correctness, as defined in other quality models [10]. If software does not provide the required functionality, then it may be reliable, portable etc., but no one will use it.

Efficiency is a characteristic that captures the ability of a correct software product to provide appropriate performance in relation to the amount of resources used. Efficiency can be considered an indication of how well a system works, provided that the functionality requirements are met. The reference to the amount of resources used, which appears in this definition is important, as the traditional measures of efficiency, such as the response time and throughput, are in fact system-level attributes.

Usability is a measure of the effort needed to learn and use a software product for the purpose chosen. The scope of this factor includes also the ease of assessment whether the software is suitable for a given purpose and the range of tolerance to the user errors. The features that are important within the context of usability are adequate documentation and support, and the intuitive understandability of the user interface.

Reliability is defined as the ability of software to maintain a specified level of performance within the specified usage conditions. Such a definition is significantly broader than the usual requirement to retain functionality over a period of time, and emphasizes the fact that functionality is only one of the elements of software quality that should be preserved by a reliable software product.

Maintainability describes the ease with which the software product can be analyzed, changed and tested. The capability to avoid unexpected effects from modifications to the software is also within the scope of this characteristic. All types of modifications, i.e. corrections, improvements and adaptation to changes in requirements and in environment are covered by this characteristic.

Portability is a measure of the effort that is needed to move software to another computing platform. This characteristic becomes particularly important in case of an application that is developed to run in a distributed heterogeneous environment or on a high performance computing platform, which lifespan is usually short. It is less important if the application runs in a stable environment that is not likely to be changed.

It can be noted from the above enumeration that the characteristics correspond to the product only and avoid any statement related to the development process. Each quality characteristic is very broad and therefore it is subdivided into a set of attributes. This quality model can be applied in the industry through the use of related 
metrics. There are 79 internal metrics defined in [4]. The metrics are quantitative and in nearly all cases take the form of a proportion: A number of functions/items/data formats/etc. that posess certain feature, related to the the number of all functions/ items/data formats/etc. that have been defined. Despite such a mathematical definition, the evaluation of each metric is a bit subjective, because whether or not a particular function/item/data format/etc. posesses certain feature is judged by the evaluator.

\section{Quality Evaluation Method}

Quality evaluation methods described in the literature [6-12], represent the software development organization point of view. The values of measures collected from particular projects create a corporate memory that can help in resolving the tasks of the project planning, implementation and evaluation. Evaluation of measures can also help during the course of a project to asses the project progress and to improve those quality characteristics that are particularly important in the context of this project.

One of the most pragmatic ways to develop the set of metrics appropriate to the project is Goal Question Metric (GQM) approach described for the first time in [6] and developed since that time by NASA. GQM provides a method for transferring business goals of the development organization into a set of measurable characteristics of a software product, process or resource. The method works in a top down manner and consists of the following three steps:

1. Define business goals, typically to improve an aspect of the development.

2. For each goal define a set of questions that must be answered in order to judge whether the goal is achieved.

3. For each question define a set of metrics that provide an appropriate information for answering the question.

The steps of selecting the questions and metrics are focused on fulfilling the specific goal that defines the context for all further activities.

Quality evaluation process, which is described in this paper, was done on behalf of the customer. Planning the process we found a great difference between the quality evaluation made for and by a software manufacturer and the evaluation that was made for the customer. One difference was such that the customer had only limited access to the project data, and the quality evaluation had to be based on an evaluation of the deliverables of the software process that had been enumerated in the contract. Another difference was such that the customer had no historical data related to a set of similar projects and could not compare the actual data to the historical one. Therefore, the customer had no specific business goals, such as to improve the software process, to use less resources or to enhance the (yet unknown) software efficiency. After signing a contract it was the manufacturer who was responsible for developing the software, while the customer wanted only to be sure that everything was done right. The rationale that stood behind such a thinking was based on a hope that if things were done right, than the results would also be right. The customer was also not able (and not willing) to answer the question, which features should be evaluated. The answer we received to such a question was always the same: Check everything. 
In order to fulfill the demands of our customer we had to change the top level of the GQM measurement model and replace the specific goals by a set of general subject areas that covered the development process and the set of deliverables from the process, as fully as possible. This way we decomposed the problem space into six subject areas. The first subject area referred to the development process itself, the next four areas corresponded to particular activities within the process, and the last one dealt with the software documentation. The following subject areas were defined:

1. Software process and development methods.

2. The analysis and analysis products.

3. The design and design products.

4. The implementation and the code.

5. Testing process and test documentation.

6. User manuals.

It can be noted from the above list that the areas $2-5$ cover all the major activities (not necessarily phases) that have been identified in both: waterfall and incremental models of software development. The decomposition of the software process into the subject areas is then exhaustive with respect to the major development activities.

The evaluation of quality within a particular subject area was decomposed into an evaluation of a set of criteria, each of which defined a specific scope of judgment. Each criterion consisted of a set of closely related questions. These questions referenced object(s) within the subject area and characterized the quality issue evaluated under this criterion. The questions were answered by metrics, i.e. data that characterized the methods used to conduct the software process or the deliverables from a particular step of the software process. To avoid problems with the interpretation of data, the sets of questions were limited to the ones that could be meaningful to the customer.

A hierarchical model of the quality evaluation is shown in Figure 1.

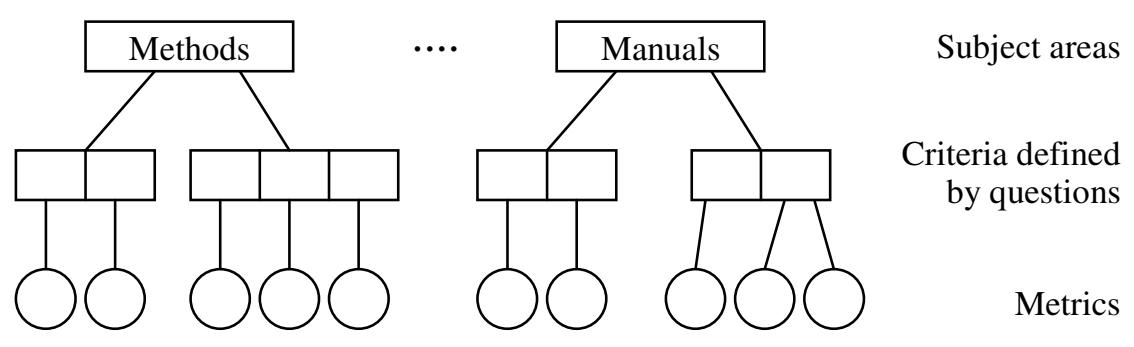

Fig. 1. Hierarchical structure of the quality evaluation model

Negative evaluation of a criterion was reported to the customer as a potential risk to the project. An advice on how to eliminate the risk was also reported to the customer as our recommendation. 


\section{Criteria, Questions and Metrics}

In order to structure the evaluation process, we organized the set of criteria within each subject area along traceability paths: From requirements origins to the requirements specification, from the requirements specification to the design, from the design to the implementation, and from the requirements specification to the test plan. Answers to the questions within a criterion were based on objective or subjective metrics. Sample criteria, questions and metrics that were defined in particular subject areas, are discussed briefly in the next three subsections. The mechanics of the quality evaluation is described in Subsection 5.4.

\subsection{Software Process and Development Methods}

The main goals of work within this subject area are the identification of methods and standards that were used throughout the development process and the evaluation of how these methods were used with respect to completeness, readability and traceability of the resulting products. In order to achieve these goals we defined the following set of criteria, accompanied by the appropriate sets of questions and metrics.

Criterion M1. Methods and standards.

Questions. Which methods (standards) are used in the development process? How is the scope of these methods? Are the methods adequate in the context of this project?

Metrics. List of methods declared for the project. A mapping from the steps of the software process into the set of methods. Evaluation of the adequacy of methods.

Criterion M2. Completeness of results.

Questions. Which artifacts recommended by the methods were created? Is the set of created artifacts sufficient? Are the results documented properly?

Metrics. List of artifacts. Evaluation of the set of artifacts. A mapping from the artifacts to the volumes of documentation.

Criterion M3. Quality of the documents.

Questions. Are the created documents readable? Are the documents consistent and unambiguous? Are the documents modifiable?

Metrics. Evaluation of readability. Evaluation of consistency. Evaluation of modifiability.

Criterion M4. Traceability of the documentation.

Questions. Is the change history of the documents maintained properly? Are the subsequent documents related to the antecedent ones?

Metrics. Evaluation of the change history (versioning). Traceability graph. Evaluation of traceability between the documents.

As can be seen from the above list of criteria, the evaluation within this subject area was focused on rather formal aspects of the software process in that it did not include an in depth analysis of the contents of the documents created throughout the development process. Nearly half of the metrics were objective, which means that they depended only on the properties of objects. Other metrics, in general those that 
began with the word "Evaluation", were subjective, which means that they depended not only on the properties of objects, but also on the viewpoint from which the evaluation was done.

The criteria correspond to the recommendations of ISO 9001, which state that a software process shall be developed, the outputs of particular activities shall be defined, and the results shall be recorded. The criteria have also a clear relation to the quality characteristics defined in ISO 9126, because the completeness of results together with readability, consistency, modifiability and traceability of the documentation promotes the maintainability and portability of the software product.

\subsection{Analysis and Analysis Products}

The main goal of work within this subject area is the evaluation of how the analysis methods were used in the project with respect to completeness, correctness and verifiability of the resulting products. There are two bunches of methods that are commonly used to perform analytical activities within the development process: Objectoriented and structured methods. Because the deliverables of both types of methods are significantly different, the quality evaluation method must be tailored to the actual analysis methodology that is used in a particular project.

The analysis performed within the IACS project was object-oriented, and relied on the use case method applied within a two-step process. In the first step, business actors and procedures were identified, and the scenarios, together with the pre- and postconditions of these procedures were defined and documented. In the second step business procedures were refined and decomposed into sets of user functions that were to be implemented by the system. A specification of a user function included a set of alternative scenarios, a definition of exceptions and exceptional actions, and the conditions to start this particular function. The structure of data that was identified within the application domain was modeled by means of a class diagram notation.

In order to evaluate the quality of analysis and analysis products we defined the following set of criteria, accompanied by the appropriate questions and metrics.

Criterion A1. Completeness of data sources.

Questions. Which sources of information were used throughout the analysis? Was the selection of law regulations complete?

Metrics. List of sources, particularly EU and national acts, cited in the analysis documents. Evaluation of completeness.

Criterion A2. Consistency between the business model and the data sources.

Questions. Is the business model consistent with law regulations that have been identified in criterion A1?

Metrics. A mapping from the set of business procedures to the set of EU and national acts. Evaluation of consistency.

Criterion A3. Completeness of the context definition.

Questions. Is the set of input data sufficient to achieve the business goals? Is the set of output data complete with respect to business and law requirements?

Metrics. A mapping from the set of goals identified in EU and national acts to the set of data (documents) input to the business procedures. Evaluation of sufficiency. A 
mapping from the set of reports defined in EU and national acts to the set of documents yielded by the business procedures.

Criterion A4. Completeness of the analysis model.

Questions. Are all of the business goals covered by the business procedures? Does the set of scenarios of each business procedure cover all types of input data?

Metrics. A mapping from the set of goals identified in EU and national acts to the set of business procedures. Evaluation of the sets of scenarios.

Criterion A5. Completeness of the functional requirements.

Questions. Are all the business procedures supported by sets of the user functions? Are all the scenarios of each business procedure covered by a set of user functions?

Metrics. A mapping from the set of business procedures to the sets of user functions. Evaluation of completeness of the coverage.

Criterion A6. Correctness of the data model.

Questions. Is the data model consistent and complete with respect to the law regulations? Does the data model comply with the best engineering practices?

Metrics. A mapping from the set of data records identified in EU and national acts to the set of classes. Evaluation of the correctness and quality of class diagrams.

Criterion A7. Usability of the user interface prototype.

Questions. Is the prototype complete? Is the prototype ergonomic?

Metrics. A mapping from the set of user functions to the sets of prototype functions. Evaluation of the ergonomics.

Criterion A8. Completeness of the non-functional requirements.

Questions. Are the non-functional requirements complete in that they define the expectations related to the security of data, performance and reliability?

Metrics. Evaluation of the non-functional requirements.

Criterion A9. Verifiability of the non-functional requirements.

Questions. Are the non-functional requirements defined in a verifiable (testable) way? Metrics. Enumeration of these requirements that are defined in a quantitative way and those that are defined qualitatively. Evaluation of the above lists.

Criterion A10. Credibility of the verification.

Questions. Which methods of verification are used? How credible is the functional part of the test plan? How credible is the non-functional part of the test plan?

Metrics. List of verification methods. Coverage of the set of all scenarios defined within the business procedures by the test scenarios. A mapping from the set of nonfunctional requirements identified in criterion A7 to the set of test scenarios. Evaluation of the non-functional tests.

As can be seen from the above list of criteria, questions and metrics, the evaluation within this subject area is focused on the contents of the analytical products. The sequence of criteria moves along a path: From sources of information to business model, from business model to functions and efficiency, from functions and efficiency to verification and validation. The set of criteria is in good relation to the set of 
quality characteristics defined in ISO 9126. The correspondence between the two is shown in Table 1. Portability is not included in the table, because this feature was not considered a significant premise in the environment of IACS project.

Table 1. A relation between the criteria and the quality characteristics of ISO 9126

\begin{tabular}{lll}
\hline Quality characteristic & Criteria & \\
\hline Functionality & A1 .. A7 & \\
Efficiency & A8, A9 \\
Usability & A7 & \\
Reliability & A8 ... A10 (also M1) \\
Maintainability & A4 ... A6 (also M2 ... M4) \\
\hline
\end{tabular}

\subsection{Testing Process and Test Documentation}

The main goal of work within this subject area is to assess the credibility of the acceptance testing of the software product. Such an evaluation must cover two different aspects of the testing process:

- The quality of the test documents, i.e. test plans, test scenarios, test cases, test data and test procedures.

- The quality of the actual execution of testing with respect to the test documents and testing standards.

Acceptance testing is the process which relates the actual characteristics of the final software product to the requirements that have been stated during the requirements analysis. Therefore, the detailed structure of the test documents depends on the way in which the requirements were defined and formulated. This makes the questions and metrics that can be used for evaluation purposes also dependent on the type of the analysis methods that were used in a particular project.

The analysis of the IACS system relied on the use case method, which was applied within a two-step process of business procedures definition and user functions definition (Section 5.2). The results of the analysis were presented in the form of quite a big set of business procedures, each of which was supported by a set of user functions. The structure of the test documents reflected this structure of the analysis products.

A basic unit of testing was an application, defined as a functional module, which provided the functionality that supported a small set of closely related business procedures. The method of testing an application was defined by a test plan, which consisted of a set of test scenarios and a test procedure. A test scenario covered a single scenario of a business procedure, and consisted of a set of steps, each of which was defined by a single test case. A test case covered a scenario or a set of scenarios of a single user function, and consisted of a set of steps that corresponded to the steps of a function scenario. There was a collection of test data and a list of acceptance criteria defined for each test case.

The questions and metrics that we defined, related test scenarios and test cases to business procedures and user functions implemented by the software. 
Criterion T1. Consistency of the test documents.

Questions. Is the structure of the test documentation consistent with the structure of analysis products and with the guidelines defined in the analysis documents?

Metrics. List of test documents. Evaluation of completeness of the test documents and the consistency between the test documents and the analysis products.

Criterion T2. Completeness of the functional testing.

Questions. Are all the functional requirements covered by the tests? Are all the user errors tested?

Metrics. A mapping from the set of business procedures to the set of test scenarios. A mapping from the set of user functions to the set of test cases. A mapping from the set of function scenarios to the set of test data. Coverage of business procedures by test scenarios. Coverage of user functions by test cases. Coverage of function scenarios by test data.

Criterion T3. Completeness of the non-functional testing.

Questions. Are all the non-functional requirements covered by the tests? Are the performance and stress tests included into the test plan?

Metrics. A mapping from the set of non-functional requirements (such as response times, throughput, re-start and data recovery times etc.) to the set of test scenarios. Evaluation of the test scenarios. Coverage of non-functional requirements by test data.

Criterion T4. Credibility of the acceptance criteria.

Questions. Are the acceptance criteria defined for each test data? Are the sets of acceptance criteria properly defined for each test case?

Metrics. A mapping from the set of test data to the set of acceptance criteria. A mapping from the set of test cases to the set of acceptance criteria. Evaluation of the acceptance criteria for each test case.

Criterion T5. Credibility of the testing process.

Questions. Is the test environment defined and documented properly? Is the test environment consistent and compatible with the production environment? Are the test results recorded properly? Are all the test scenarios defined in the test plan executed during the testing process?

Metrics. Evaluation of the test environment documentation. A mapping from the production environment definition (list of elements) to the test environment definition. Observation of the testing process and comparison with the test reports. A mapping from the set of test scenarios to the set of test reports. Evaluation of the test reports.

The set of evaluation criteria defined above relates to a subset of quality characteristics defined in ISO 9126. The correspondence between the two is shown in Table 2 . The other three characteristics of ISO 9126 are not included in the table, because they were not verified by our team. Usability of the software was verified during the testing process directly by the testers that represented our customer. Maintainability could not be verified by means of testing. Portability was not considered a significant premise in the environment of IACS project. 
Table 2. A relation between the criteria and the quality characteristics of ISO 9126

\begin{tabular}{ll}
\hline Quality characteristic & Criteria \\
\hline Functionality & T1, T2, T4, T5 \\
Efficiency & T3, T4, T5 \\
Reliability & T3, T4, T5 \\
\hline
\end{tabular}

The answers given by metrics to the questions stated above created the basis for a final recommendation on whether or not to accept the software product.

\subsection{Evaluation Process}

The software for IACS system was developed iteratively according to the guidelines of RUP - Rational Unified Process [13]. Input data to the process of quality evaluation consisted of all the documents that were created in the entire software development cycle. These included:

- Business model developed in the inception phase.

- Early analysis model of the elaboration phase.

- The set of analysis and design models created in the construction phase.

- The code and the complete set of manuals.

- Test plans and test reports (plus the observation of the testing process).

Because of the incremental nature of the development, part of the documents circulated in several versions, issued in a sequence of subsequent increments. The evaluation of the deliverables form particular increments of the process dealt mainly with new documents and other products, however, the scope of changes to the products delivered in the previous increments was also subject to investigation.

The evaluation process was decomposed into the set of subject areas listed in section 4 and was structured according to the set of criteria exemplified in sections 5.1 through 5.3. The evaluation that was done within the context of a particular criterion was guided by the set of questions and metrics.

The scope of criteria related to development methods, described in Section 5.1, was very broad, because it related to all the phases and activities of the entire development process. Therefore the evaluation of these criteria was decomposed in such a way that the questions were stated and answered separately for particular groups of artifacts. For example, the evaluation of criterion M1 (Methods and standards) was decomposed into an investigation of the software process, the analysis methods, the design methods, the implementation methods and tools, the testing methods and the documentation standards.

The answers were given to questions by metrics, only few of which were quantitative, i.e. evaluated to a numerical value. However, many metrics were formal, i.e. took the form of a mapping between the sets of artifacts or documents. Internal metrics of ISO 9126, defined as proportions, appeared useless within the environment of this project, particularly within the area of analysis. The customer required full coverage and correctness in that all the identified requirements had to be reflected in the analysis model, all the business procedures had to be specified and supported by user functions, all the data types had to be serviced, etc. $90 \%$ was not very different from 
zero. An exception was the area related to testing and documentation, in which the metrics of coverage were used.

Answers to questions within a particular criterion were aggregated into a general mark within the scale of: good, satisfactory, bad, dangerous. The two lowest marks were reported to the customer as risks. The evaluation report was structured in accordance with subject areas and criteria. The results of the evaluation within a particular subject area were concluded in the form of two sections: Risks for the project and recommendations to the project. The risk section reported the bad and dangerous marks given to particular criteria within this subject area, related to the quality characteristics of ISO 9126. For example:

- The lack of functions that support certain business procedures creates the risk that the required functionality of software will not be met.

- The lack of readable design models creates the risk that the maintainability of software will be unacceptable low.

The recommendation section advised on what to do in order to avoid the risks identified in the evaluation process and described in the previous section. Recommendations related to the risks listed above could read, e.g.:

- Define the functionality that is missing.

- Create the models that are missing and improve readability of those that exist.

\section{Conclusions}

This paper describes a practical method that can be used to evaluate the expected quality of software under design. The evaluation process does not refer directly to the existing standards, however, it is consistent with the definitions of the quality models of both ISO 9001 and ISO 9126. The mechanics of the evaluation is based on a set of criteria that are decided by stating questions and finding answers to those questions. The collection of criteria is structured into a set of subject areas that cover the set of activities or phases that exist in the most popular software processes.

The method was used successfully in evaluating the expected quality of software developed for a huge governmental system. The evaluation was performed on behalf of the customer and not the manufacturer of the system. The criteria, questions and metrics that helped in answering the questions allowed for a systematic, in depth analysis of the deliverables of the particular development activities. As result, several risks that could have a negative impact on the quality of the resulting software were revealed and identified. The recommendations helped the customer in avoiding these risks. IACS system was build and certified for use within the deadline.

The advantages of the method can be summarized as follows:

- The method can be tailored to any particular software process or method that can be used in the development of software.

- The application of the method leads to such results, i.e. to the evaluation of criteria, that are readable and meaningful to the customer. 
- Negative evaluation of particular criteria can easily be translated into risk warnings and recommendations on what to improve in order to enhance the expected quality of the final product.

The method is simple in use, does not relay on any historical data, and need not be supported by a computerized tool.

\section{References}

1. ISO 9001: Quality management systems - Requirements. ISO (2001)

2. ISO/IEC 9126-1: Software engineering - Product quality - Part 1: Quality model. ISO/IEC (2001)

3. ISO/IEC TR 9126-2: Part 2: External metrics. ISO/IEC (2001)

4. ISO/IEC TR 9126-3: Part 3: Internal metrics. ISO/IEC (2001)

5. ISO/IEC TR 9126-4: Part 4: Quality in use metrics. ISO/IEC (2001)

6. Basili, V.R., Weiss, D.M.: A Methodology for Collecting Valid Software Engineering Data, IEEE Trans. Software Eng., 6 (1984) 728-738

7. Basili, V.R., Caldiera, G., Rombach, H.D.: The Goal Question Metric Approach. In: Encyclopedia of Software Engineering, Wiley-Interscience, New York (1994)

8. Solingen, R., Berghout, E.: The Goal/Question/Metric Method, McGraw-Hill (1999)

9. Erikkson, I., McFadden, F.: Quality Function Deployment: A Tool to Improve Software Quality. In: Information \& Software Technology, 9 (1993) 491-498

10. Fenton, N: Software Metrics: A Rigorous Approach, Chapman and Hall (1993)

11. Haag, S., Raja, M.K., Schkade, L.L.: Quality Function Deployment Usage in Software Development. In: Communications of the ACM, 1 (1996) 41-49

12. Lethbridge, T.C., Sim, S.E., Singer, J.: Studying Software Engineers: Data Collection Techniques for Software Field Studies, Empirical Software Engineering, 10 (2005) 311341

13. Kruchten, P.: Rational Unified Process: An Introduction, Addison Wesley (2003) 